\title{
Resilience acts as a moderator in the relationship between infertility-related stress and fertility quality of life among women with infertility: a cross-sectional study
}

Yifei $\mathrm{Li}^{1+}$, Xin Zhang ${ }^{1+}$, Meng Shi ${ }^{2}$, Shuaishuai Guo ${ }^{3{ }^{*+}}$ and Lie Wang ${ }^{1 *+}$ (D)

\begin{abstract}
Background: Infertility-related stress can seriously impact the fertility quality of life (QoL) of infertile women. Resilience, as a coping resource, can effectively combat psychological stress. This study aimed to evaluate the fertility QoL of infertile women and to examine whether resilience moderates the association of infertility-related stress with fertility QoL.

Methods: This cross-sectional study was conducted in northeast of China from December 2017 to February 2018. Out of 559 women outpatients with infertility, 498 (89.1\%) completed self-reported questionnaires including the FertiQoL Scale, Fertility Problem Inventory (FPI) and Connor-Davidson Resilience Scale (CD-RISC). Hierarchical multiple regression analysis and simple slope analysis were applied to explore the influencing factors related to fertility QoL as well as to examine the moderating effect of resilience on the association of infertility-related stress with fertility QoL.

Results: The mean FertiQoL score was $64.54 \pm 16.90$ among the participants. Household monthly income and causes of infertility were significantly related to fertility QoL. In addition, infertility-related stress was negatively related to fertility QoL, and resilience was positively associated with fertility QoL, explaining 36.3\% of the variance. Resilience moderated the association of infertility-related stress with fertility QoL. Specifically, the effect of infertility-related stress on fertility QoL varied by low(1 SD below the mean, $B=-0.496, \beta=-0.714, P<0.001)$, mean $(B=-0.293, \beta=-0.422$, $P<0.001$ ) and high (1 SD above the mean, $B=-0.090, \beta=-0.130, P<0.001$ ) levels of resilience. The higher resilience was, the weaker the effect of infertility-related stress on fertility QoL was.

Conclusions: Overall, women with infertility in China had relatively low FertiQoL scores. Resilience influenced the association of infertility-related stress with fertility QoL. Infertile patients' psychological status must be addressed and adequate resilience-based interventions such as mindfulness-based skills should be provided to improve fertility QoL of women with infertility.
\end{abstract}

Keywords: Infertility, Quality of life, Infertility-related stress, Resilience, Moderating role

\footnotetext{
* Correspondence: shuaishuai197411@sina.com; liewang@cmu.edu.cn

${ }^{\dagger}$ Yifei Li, Xin Zhang, Shuaishuai Guo and Lie Wang contributed equally to this

work.

${ }^{3}$ Reproductive Medicine Center, Shenyang Women's and Children's Hospital,

No.87 Danan Road, Shenhe Area, Shenyang 110000, China

${ }^{1}$ Department of Social Medicine, School of Public Health, China Medical

University, No.77 Puhe Road, Shenyang North New Area, Shenyang 110122,

China

Full list of author information is available at the end of the article
}

(c) The Author(s). 2019 Open Access This article is distributed under the terms of the Creative Commons Attribution 4.0 International License (http://creativecommons.org/licenses/by/4.0/), which permits unrestricted use, distribution, and reproduction in any medium, provided you give appropriate credit to the original author(s) and the source, provide a link to the Creative Commons license, and indicate if changes were made. The Creative Commons Public Domain Dedication waiver (http://creativecommons.org/publicdomain/zero/1.0/) applies to the data made available in this article, unless otherwise stated. 


\section{Background}

According to Zegers-Hochschild et al., the latest definition of infertility is "a disease characterized by the failure to establish a clinical pregnancy after 12 months of regular, unprotected sexual intercourse or due to an impairment of a person's capacity to reproduce either as an individual or with his/her partner" [1]. Worldwide, infertility is a prevalent condition among women aged 15 to 49 years. Previous studies have reported that $15.5 \%$ of reproductive-age women in the United States suffer from infertility [2], as well as $24 \%$ in France [3] and $25 \%$ in China [4]. Infertility can lead to various physical, psychological and societal consequences, such as depression, anxiety, stigma and social isolation, which may significantly impact patients' fertility quality of life (QoL) [5-7].

QoL is defined by the World Health Organization (WHO) as individuals' perceptions of their position in life in the context of culture and the value systems where they live [8]. Accordingly, fertility QoL involves a reflection of infertile patients' life status during their infertile period in a broad sense. A large number of studies revealed that compared with the fertile counterparts, infertile women experienced poorer QoL during the period of infertility [9-11]. In addition, poor fertility QoL of infertile women was shown to be negatively related to treatment compliance and could cause latent economic burdens on their families and communities [12]. However, the identification of the influencing factors of fertility QoL makes it possible to conduct targeted interventions and care activities in an integrated way to improve the fertility QoL of women with infertility, which necessitates our study.

The impact of infertility-related stress on fertility QoL is an increasingly important topic in recent scientific research. Infertility-related stress refers to perceived stresses from one's social networks, marital relationships, and physical and mental health due to infertility [13]. In many societies, especially in China, infertility and the consequent childlessness are often correlated with stigma and guilt $[6,14]$. As a result, infertile women may have a strong sense of loneliness and social stress. In addition, Ganth et al. found that most infertile couples were dissatisfied with their marital lives, which could predispose women to additional marital stress [15]. Furthermore, it is well accepted that both a diagnosis and infertility treatment can induce heavy physical and psychological stress [16]. Several well-documented studies have indicated that infertile women seem to carry a much heavier burden and stress than their male counterparts [17, 18]. Therefore, women with infertility may perceive considerable infertility-related stress that can result in a life crisis and significantly impact their fertility QoL.
As a positive psychological resource, resilience has attached increasing importance in clinical practice. Resilience is defined as the developable capabilities to rebound or bounce back from tragedy, frustration and failure or even positive events [19]. Resilient patients are usually considered to possess self-esteem, believing in one's own self-efficacy and having effective problem-solving skills to cope with stress $[20,21]$. Many previous studies have demonstrated that resilience has direct and positive effects on patients' QoL $[22,23]$. Specifically, resilience was found to be strongly and positively related to QoL among women with infertility [24]. In addition, prior studies have supported the mediating effect of resilience resources on QoL $[22,25]$. Resilience can also act as a moderator to buffer the effect of antecedent indicators on QoL. For example, Palm-Fischbacher et al. reported that resilience could act as a moderator in the association of chronic stress with physical health among young women [26]. Another study by Rainone et al. showed that resilience could moderate the relationship between affective disorders and QoL among patients with multiple sclerosis [27]. Since the protective effect of resilience on QoL and its negative effect on perceived stress have been widely reported [22-28], resilience seems to be able to moderate the association of infertilityrelated stress with fertility QoL. In other words, the effect of infertility-related stress on the fertility QoL can be affected by the levels of resilience patients possess.

However, to the best of our knowledge, extant studies have not yet explored resilience as a moderator in the relationship between infertility-related stress and fertility QoL among women with infertility. Therefore, in the present study, we aimed to evaluate the fertility QoL among these patients to examine whether resilience moderates the association of infertility-related stress with fertility QoL and to find solutions to improve fertility QoL.

\section{Methods \\ Ethics statement}

The study protocol was in accordance with the ethical standards and was approved by the Ethics Committee of Shenyang Women's and Children's Hospital. Written informed consent was obtained from each participant. Information collected from all participants was kept confidential and anonymous.

\section{Study design and data collection}

This cross-sectional study was carried out in outpatients diagnosed with infertility from December 2017 to February 2018. All participants were recruited at the Reproductive Medicine Center of Shenyang Women's and Children's Hospital in Liaoning Province, northeastern China. The inclusion criteria were as follows: 1) women outpatients diagnosed with infertility and aged over 18 years; 2) women 
who received in vitro fertilization and embryo transfer (IVF-ET) treatment; 3) women who were literate and could communicate well in Chinese; and 4) women who were willing to voluntarily complete a multi-item questionnaire. The exclusion criteria included the following: 1) women who had other major diseases (such as cancers and severe cardiovascular and cerebrovascular diseases) at the present stage; 2) women who had a psychiatric history; and 3) women who had intellectual and/or cognitive impairments. After obtaining their written informed consent for this study, a self-reported questionnaire was distributed to each eligible participant, and clinical data were collected from their medical records. Questionnaires with any missing data were excluded from statistical analyses. Finally, out of the 559 eligible participants, 27 patients declined to participate and 34 questionnaires were excluded. In total, 498 complete responses were received $(89.1 \%)$ in the present study.

\section{Measures}

\section{Measurement of fertility QoL}

We used the Chinese version of the FertiQoL Scale to measure fertility QoL in infertile women [29]. The Chinese version of the FertiQoL Scale has been translated from the international FertiQoL questionnaire and consists of two modules: a core FertiQoL module and an optional treatment module [30]. The optional FertiQoL treatment module was not used in this study. The Chinese version of the core FertiQoL module contained two general items and 22 specific items covering mind-body, relational, social and emotional domains (e.g., Do your fertility problems interfere with your day-to-day work or obligations?). Each item was rated on a five-point Likert scale from 0 to 4 . The total raw scores were computed and transformed to standard scores ranging from 0 to 100 , with a higher score reflecting a higher fertility QoL. The Chinese version of the FertiQoL scale has been widely used and has shown good reliability and validity among Chinese populations [29, 31]. In present study, the Cronbach's alpha coefficient of the FertiQoL Scale was 0.925 .

\section{Measurement of infertility-related stress}

The Fertility Problem Inventory (FPI) compiled by Newton et al. is a useful tool to measure the level of infertilityrelated stress in five dimensions, namely, social concern, relationship concern, sexual concern, the need for parenthood, and rejection of a child-free lifestyle [32]. In this study, the infertility-related stress of the participants was measured with the Chinese version of FPI, which comprises 46 items (e.g., I can't help comparing myself with friends who have children) [33]. Each item was scored using a six-point Likert scale ranging from 1 (do not agree) to 6 (totally agree). The overall scores ranged from 46 to 276 , and a higher score indicated higher perceived fertility-related stress. The Chinese version of the FPI was reported to have adequate reliability and validity [33]. The Cronbach's alpha coefficient of FPI was 0.823 in this study.

\section{Measurement of resilience}

The Chinese version of the widely used Connor-Davidson Resilience Scale (CD-RISC), developed by Connor et al., was used to assess resilience [34, 35]. The Chinese version of CD-RISC is a 25-item scale (e.g., coping with stress strengthens me) and the items are scored on a five-point scale ranging from 0 (not true at all) to 4 (true nearly all the time). A sum score was calculated with higher total scores reflecting higher levels of resilience. The Chinese version of CD-RISC has shown adequate reliability and validity $[35,36]$. The Cronbach's alpha coefficient of the Chinese version of CD-RISC was 0.943 in this study.

\section{Demographic characteristics}

Demographic characteristics included age, educational background, residence and household monthly income. Educational background was categorized into senior high school or below, junior college and college or above. Residence was classified into rural and urban areas. Household monthly income was divided into $\leq 4000$ and $>4000$ yuan (approximately 600 dollars).

\section{Clinical variables}

Four clinical variables were measured including pregnancy history, surgical history, infection history and causes of infertility. Pregnancy history and surgical history were categorized into yes or no answers. Infection history was defined as "yes" if the respondents had suffered from sexually transmitted infections or had a history of induced abortion infection; otherwise, it was categorized as "no". The causes of infertility were divided into three groups: female factors (e.g., ovulation disorders, endometriosis), mixed factors (female factors mixed with male factors such as oligospermia, erectile dysfunction, etc.) and unexplained reasons.

\section{Statistical analysis}

The mean scores of FertiQoL with different categories of demographic and clinical variables were examined by Student's $t$-test or one-way ANOVA. Pearson's correlation analysis was used to analyze the correlation among fertility QoL, infertility-related stress and resilience. Hierarchical multiple regression analysis was applied to investigate the factors in relation to fertility QoL as well as to explore the moderating role of resilience on the association of infertility-related stress with fertility QoL. All variables that were associated with fertility QoL in univariate analysis $(P<0.05)$ were entered into the hierarchical multiple regression model. In the model, age and potential control variables were entered in step 1 . 
Infertility-related stress and resilience were added in step 2. Finally, the product of infertility-related stress and resilience was added in step 3 . The hypothesis of the moderating effect of resilience was supported if the interaction was significant, and simple slope analysis was conducted to visualize the interaction term. The variables in the model were centralized before regression analysis was conducted. All the statistical analyses above were performed with SPSS for Windows (version 20.0), with a two-tailed $P$-value of $<0.05$ considered to be statistically significant.

\section{Results}

\section{Description of the participants and fertility QoL}

Demographic and clinical characteristics and group differences in fertility QoL are presented in Table 1. The age range of participants varied from 19 to 40 years (mean: $32.19 \pm 3.83$ ). The proportion of the participants with an education background level at senior high school or below was $39.6 \%$ (197); $77.5 \%$ (386) of the participants lived in urban areas. There were 309 (62.0\%) participants who had a household monthly income exceeding 4000 yuan. With regard to clinical variables, $45.8 \%$ (228) and $41.2 \%$ (205) of the participants had a history of pregnancy and surgical history, respectively. Only $7.2 \%$ (36) of the participants had an infection history. There were 299 (60.0\%), 56 (11.3\%) and 31 (6.2\%) patients suffering from infertility because of female factors, male factors and mixed factors, respectively. Participants whose monthly income exceeded 4000 yuan experienced a higher level of fertility QoL compared with patients whose household monthly income was below 4000 yuan $(t=2.446, P=0.015)$, and the patients with infertility due to female factors reported lower fertility QoL than the other groups $(t=4.079, P=0.007)$.

Table 1 FertiQoL scores by demographic and clinical characteristics

\begin{tabular}{|c|c|c|c|c|c|c|}
\hline \multirow[t]{2}{*}{ Variables } & \multirow[t]{2}{*}{$\mathrm{n}$} & \multirow[t]{2}{*}{$\%$} & \multicolumn{4}{|c|}{ FertiQoL } \\
\hline & & & $\overline{\text { Mean }}$ & SD & $F / t$ & $P$-value \\
\hline \multicolumn{7}{|l|}{ Demographic variables } \\
\hline Educational background & & & & & 0.914 & 0.401 \\
\hline Senior high school or below & 197 & 39.6 & 65.78 & 16.70 & & \\
\hline Junior college & 111 & 22.3 & 64.07 & 16.60 & & \\
\hline College or above & 190 & 38.1 & 63.53 & 17.29 & & \\
\hline Residence & & & & & 1.275 & 0.203 \\
\hline Rural area & 112 & 22.5 & 66.33 & 16.22 & & \\
\hline Urban area & 386 & 77.5 & 64.02 & 17.08 & & \\
\hline Household monthly income (yuan) & & & & & 2.446 & 0.015 \\
\hline$\leq 4000$ & 189 & 38.0 & 62.19 & 17.57 & & \\
\hline$>4000$ & 309 & 62.0 & 65.98 & 16.34 & & \\
\hline \multicolumn{7}{|l|}{ Clinical variables } \\
\hline Pregnancy history & & & & & 0.467 & 0.641 \\
\hline Yes & 228 & 45.8 & 64.16 & 15.76 & & \\
\hline No & 270 & 54.2 & 64.86 & 17.83 & & \\
\hline Surgical history & & & & & 1.874 & 0.062 \\
\hline Yes & 205 & 41.2 & 62.85 & 17.57 & & \\
\hline No & 293 & 58.5 & 65.73 & 16.34 & & \\
\hline Infection history & & & & & 1.737 & 0.083 \\
\hline Yes & 36 & 7.2 & 59.84 & 20.16 & & \\
\hline No & 462 & 92.8 & 64.91 & 16.59 & & \\
\hline Causes of infertility & & & & & 4.079 & 0.007 \\
\hline Female factors & 299 & 60.0 & 62.75 & 17.32 & & \\
\hline Male factors & 56 & 11.3 & 70.49 & 18.18 & & \\
\hline Mixed factors & 31 & 6.2 & 63.73 & 14.25 & & \\
\hline Unexplained reason & 112 & 22.5 & 66.58 & 14.98 & & \\
\hline
\end{tabular}


Descriptive statistics and correlations of continuous variables The scores of FertiQoL, age, infertility-related stress and resilience as well as the correlation coefficients between them are displayed in Table 2. The mean score of FertiQoL was 64.54 \pm 16.90 . Infertility-related stress was negatively correlated with fertility QoL $(P<0.01)$, whereas resilience was positively correlated with fertility QoL $(P<0.01)$. In addition, infertility-related stress was negatively correlated with resilience $(P<0.01)$.

\section{The moderating effect of resilience in the relationship between infertility-related stress and fertility QoL}

As shown in Table 3, hierarchical regression analysis was used to examine the moderating effect of resilience on the association of infertility-related stress with fertility QoL. In step 1, the demographic and clinical variables in the univariate analysis $(P<0.05)$ were entered as covariates, including age, household monthly income and causes of infertility. The linear combination of these control variables partially explained the variance in fertility QoL $\left(F=3.789\right.$, adjusted $\left.R^{2}=0.027, P<0.01\right)$. In step 2, infertility-related stress was found to be significantly and negatively related to fertility QoL $(B=-0.280, \beta=-0.403, P<0.01)$, while resilience was significantly and positively associated with fertility QoL $(B=0.316, \beta=0.302, P<0.01)$. Infertility-related stress and resilience exhibited significant effects on fertility QoL $\left(F=46.646\right.$, adjusted $R^{2}=0.391, \quad \Delta R^{2}=0.363$, $P<0.01)$. In step 3 , the infertility-related stress $\times$ resilience interaction term was significantly and positively associated with fertility QoL $(F=60.528$, adjusted $\left.R^{2}=0.489, \Delta R^{2}=0.098, P<0.01\right)$. Thus, resilience played a moderating role in the relationship between infertility-related stress and fertility QoL. Simple slope analysis of the interaction presented in Fig. 1, which showed that the impacts of infertility-related stress on fertility QoL were different in low (1 SD below the mean, $B=-0.496, \beta=-0.714, P<0.001)$, mean $(B=-0.293$, $\beta=-0.422, P<0.001)$ and high (1 SD above the mean, $B=-0.090, \beta=-0.130, P<0.001)$ levels of resilience; when resilience was higher, the effect of infertility-related stress on fertility QoL became weaker.

Table 2 Scores and correlations of continuous variables

\begin{tabular}{llllll}
\hline Variables & Mean & SD & 1 & 2 & 3 \\
\hline 1. Fertility QoL & 64.54 & 16.90 & 1 & & \\
2. Age & 32.19 & 3.83 & 0.048 & 1 & \\
3. Infertility-related stress & 145.57 & 24.33 & $-0.575^{* *}$ & $-0.103^{*}$ & 1 \\
4. Resilience & 59.53 & 16.18 & $0.535^{* *}$ & $0.117^{* *}$ & $-0.563^{* *}$
\end{tabular}

Note: QoL indicates quality of life; SD indicates standard deviation *indicates $P<0.05$, ** indicates $P<0.01$
Table 3 Hierarchical regression results of fertility QoL

\begin{tabular}{|c|c|c|c|c|c|}
\hline Variables & $B$ & SE $B$ & $\beta$ & $T$ & $P$-value \\
\hline \multicolumn{6}{|l|}{ Step 1} \\
\hline Age & 0.199 & 0.196 & 0.045 & 1.012 & 0.312 \\
\hline Household monthly income & 3.532 & 1.552 & 0.101 & 2.275 & 0.023 \\
\hline \multicolumn{6}{|l|}{ Causes of infertility } \\
\hline Dummy_1 & 7.832 & 2.430 & 0.147 & 3.223 & 0.001 \\
\hline Dummy_2 & 1.722 & 3.159 & 0.025 & 0.545 & 0.586 \\
\hline Dummy_3 & 3.570 & 1.850 & 0.088 & 1.930 & 0.054 \\
\hline$F$ & 3.789 & & & & \\
\hline Adjusted $R^{2}$ & 0.027 & & & & \\
\hline$P$-value & 0.002 & & & & \\
\hline \multicolumn{6}{|l|}{ Step 2} \\
\hline Infertility-related stress & -0.280 & 0.030 & -0.403 & -9.477 & $<0.001$ \\
\hline Resilience & 0.316 & 0.045 & 0.302 & 6.978 & $<0.001$ \\
\hline F & 46.646 & & & & \\
\hline Adjusted $R^{2}$ & 0.391 & & & & \\
\hline$\Delta R^{2}$ & 0.363 & & & & \\
\hline$P$-value & $<0.001$ & & & & \\
\hline \multicolumn{6}{|l|}{ Step 3} \\
\hline $\begin{array}{l}\text { Infertility-related } \\
\text { stress } \times \text { resilience }\end{array}$ & 0.013 & 0.001 & 0.317 & 9.749 & $<0.001$ \\
\hline$F$ & 60.528 & & & & \\
\hline Adjusted $R^{2}$ & 0.489 & & & & \\
\hline$\Delta R^{2}$ & 0.098 & & & & \\
\hline$P$-value & $<0.001$ & & & & \\
\hline
\end{tabular}

Note: QoL indicates quality of life, $S E$ indicates standard error; Dummy_1 indicates male factors vs. female factors; Dummy_2 indicates mixed factors vs. female factors; Dummy_3 indicates unexplained reason vs. female factors

\section{Discussion}

This study evaluated the fertility QoL and its associated factors among women with infertility in China. We recruited 559 participants with a highly effective response rate of $89.1 \%$. To the best of our knowledge, the present study was the first to explore the moderating role of resilience on the association of infertility-related stress with fertility QoL in infertile women.

We found that the mean score of FertiQoL in infertile Chinese women was $64.54 \pm 16.90$, which was much lower than findings in most previous studies across countries, such as Turkey $(66.97 \pm 14.35)$, the Netherlands $(70.80 \pm 13.90)$, the United States $(72.30 \pm$ 14.80), and Germany $(73.00 \pm 12.00)$ [37-40]. Despite the fact that there are affordable and advanced technologies, such as IVF-ET and easier ways to obtain access to better quality care in China, our results revealed that Chinese women with infertility still experienced poor fertility QoL. Thus, it is very imperative to determine the crucial influencing factors and targeted solutions to improve their QoL. 


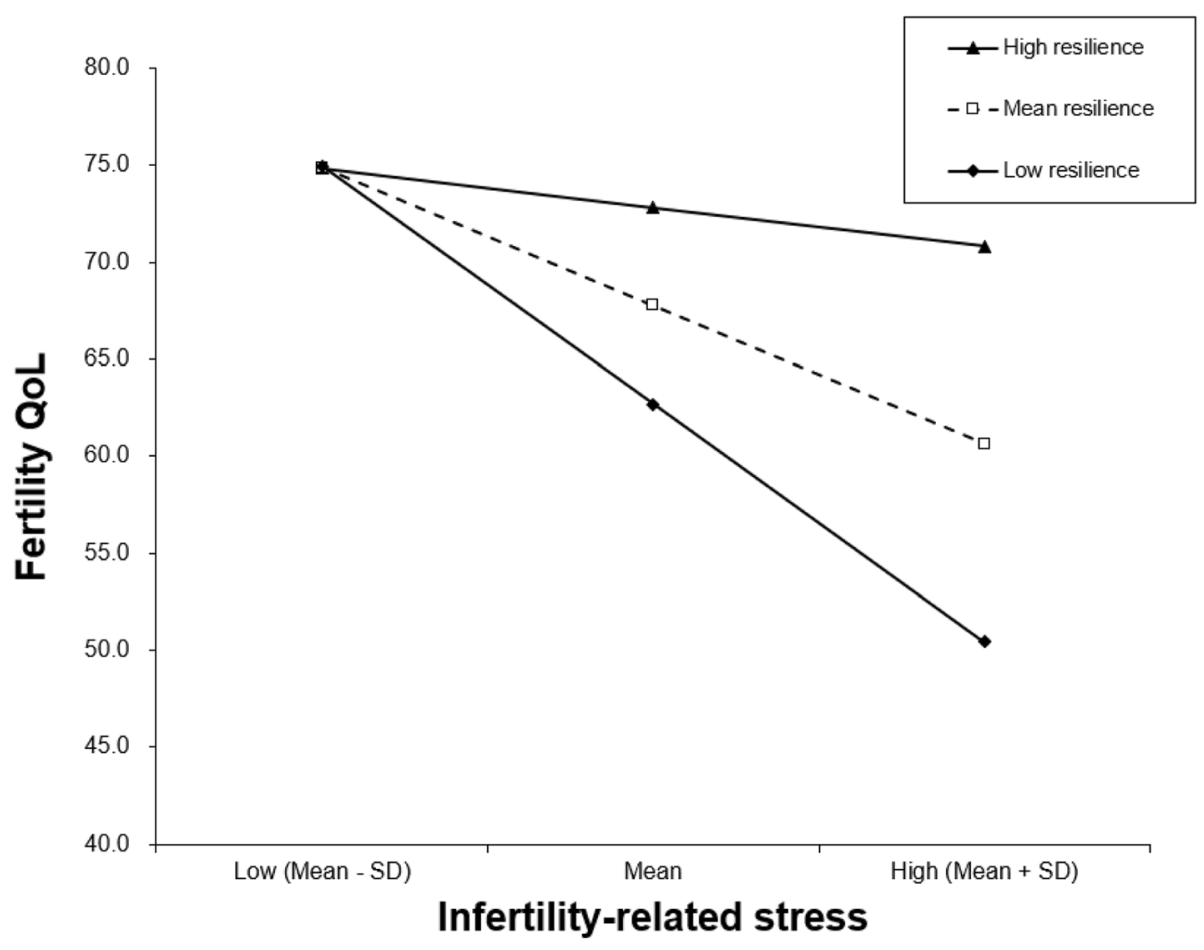

Fig. 1 Simple slope plot of the interaction between infertility-related stress and resilience on fertility QoL. Note: The values of infertility-related stress and resilience were centered before regression analysis. Age, household monthly income and causes of infertility were adjusted. QoL indicates quality of life; low indicates 1 SD below the mean; high indicates 1 SD above the mean; SD indicates standard deviation

Among the demographic and clinical variables, only household monthly income and causes of infertility were significantly related to FertiQoL score, explaining $2.7 \%$ of the variance, which echoed the findings of previous studies $[7,9,41]$. This result indicated that the reduction of treatment costs should be considered by hospital managers to lessen the financial burden of infertile patients. Moreover, as the results showed, women with infertility caused by a female factor had the lowest FertiQoL score. Thus, the medical staff and their family members ought to be encouraged to give more care and to support to them, which may be helpful in improving their fertility QoL [42].

The results of this study showed that after controlling for the covariates, infertility-related stress and resilience were key factors affecting fertility QoL, explaining 36.3\% of the total variance. Specifically, infertility-related stress was found to be significantly and negatively related to fertility QoL, which was consistent with previous studies $[18,43,44]$. Perceived stress can be considered a result of dynamic interactions between the external environment and the individual [45]. When reproductive-age women are diagnosed with infertility, this negative life event and feeling of stigma can induce heavy psychological distress. The accumulated stress can induce persistent negative emotions such as anxiety, depression and social isolation, seriously impairing patients' QoL
$[28,46]$. In addition, infertility-related stress may lead to increased marital conflicts and decreased life satisfaction between wives and husbands, which can also seriously impact their quality of marital life [15, 47]. Noticeably, the mean scores of infertility-related stress of the participants in this study were found to be higher than their counterparts in other countries [17, 48, 49], which may partly explain why Chinese infertile women experienced poorer fertility QoL. Additionally, the higher level of stress can be attributed to the differences in cultures and values. In Chinese society, maternity signifies social respectability to a large extent, whereas childlessness means unfiliality (this terminology means not respecting one's parents, elders, and ancestors). Therefore, future studies should focus more on cultural factors when identifying stressors impacting fertility QoL.

With regard to resilience, we found that it was significantly and negatively correlated with infertility-related stress and positively associated with fertility QoL, which was similar to prior studies $[24,50]$. In addition, we also found that resilience moderated the association of infertility-related stress with fertility QoL, which confirmed our hypothesis. The result of the simple slope analysis showed that the higher the resilience, the weaker the effect of infertility-related stress on fertility QoL. Infertility-related stress can lead to many negative emotions and adverse physiological responses (e.g., 
headaches) which may overwhelm patients [51], while resilient patients are usually characterized by possessing high levels of self-esteem, self-efficacy and optimism, and they can take advantage of problem-solving skills to effectively cope with stress [20, 21]. Reasonably, infertile patients with high levels of resilience enjoyed high fertility QoL because they could recover quickly and easily from challenges in both daily life and disease conditions. This result implies that resilience as a protective factor of infertile women can reduce their perceived psychological distress, which is conducive to maintaining their physical, mental and social well-being. In addition, it highlighted the contribution of the interaction between perceived stress and resilience to fertility QoL. This means that if infertility-related stressors are hard to be effectively reduced, improving resilience may be a good way to minimize the negative impact of infertility-related stress on fertility QoL.

Based on our findings, several implications for clinical practice should be highlighted to improve fertility QoL of women with infertility. First, during the diagnosis and treatment of infertility, patients' psychological status must be evaluated. Second, psychological counseling services should be provided to infertile couples [52]. Third, infertile patients need more social support from family members, clinicians and nurses. Finally, it is important to build resilience to improve fertility QoL in infertile women who are laden with considerable stress. As building resilience is usually considered a dynamic process, interventions such as mindfulness-based skill and cognitive-behavioral approaches could be introduced to infertile patients in the early disease stage in order to increase the protective effect of resilience on fertility QoL [53-56]. Patients could also regularly engage in a proactive personal reflective report to increase their resilience [57]. Overall, targeted intervention strategies should be conducted in future research.

Several limitations should be mentioned in the present study. First, a cross-sectional design was used in the study, and no causal conclusions could be drawn between the variables investigated. A longitudinal study should be carried out to verify our findings. Second, infertility-related stress, resilience and fertility QoL were measured using self-administered questionnaires, which could lead to possible recall bias or response bias. Third, several potential factors, such as beliefs about the importance of parenthood and personality, may affect QoL related to infertility and were not included in the study. Finally, this study was conducted in a province of the northeastern region of China. Thus, caution should be taken when extrapolating the results to infertile patients in other parts of China.

\section{Conclusions}

Overall, women with infertility in northeastern China experienced relatively low fertility QoL. Infertility-related stress and resilience were the crucial factors associated with fertility QoL, and resilience moderated the association of infertility-related stress with fertility QoL. Thus, in clinical practice, more attention should be paid to psychological stress, and more social support should be provided to Chinese women with infertility. More importantly, adequate resilience interventions such as mindfulness-based skills should be introduced to improve their fertility QoL.

\section{Abbreviations \\ CD-RISC: Connor-Davidson Resilience Scale; FertiQoL: Fertility quality of life; FPI: Fertility Problem Inventory; QoL: Quality of life; SD: Standard deviation; SE: Standard error; WHO: World Health Organization}

\section{Acknowledgments \\ The authors would like to thank all the patients who voluntarily participated in this study and research assistants who performed the data collection.}

\section{Funding}

There were no funding sources.

Availability of data and materials

The datasets analyzed during the current study are available from the corresponding author on reasonable request.

\section{Authors' contributions}

YFL carried out data collection, analysis, interpretation, and wrote the paper. $X Z$ performed data collection, cleaned the data and revised the paper. YFL and $X Z$ contributed equally, and are the co-first authors of this paper. LW and SSG provided guidance in the study design, organized the investigation, and are the co-corresponding authors. MS assisted with the data interpretation and revised the paper. All authors have read and approved the final version of the manuscript.

\section{Ethics approval and consent to participate}

All patients provided their informed written consent. The study was approved by the Ethics Committee of Shenyang Women's and Children's Hospital and

was conducted in accordance with the principles of the Declaration of Helsinki.

Consent for publication

Not applicable.

\section{Competing interests}

The authors declare that they have no competing interests.

\section{Publisher's Note}

Springer Nature remains neutral with regard to jurisdictional claims in published maps and institutional affiliations.

\section{Author details}

${ }^{1}$ Department of Social Medicine, School of Public Health, China Medical University, No.77 Puhe Road, Shenyang North New Area, Shenyang 110122, China. ${ }^{2}$ English Department, School of Fundamental Sciences, China Medical University, No.77 Puhe Road, Shenyang North New Area, Shenyang 110122, China. ${ }^{3}$ Reproductive Medicine Center, Shenyang Women's and Children's Hospital, No.87 Danan Road, Shenhe Area, Shenyang 110000, China. 
Received: 10 August 2018 Accepted: 24 January 2019

\section{Published online: 15 February 2019}

\section{References}

1. Zegers-Hochschild F, Adamson GD, Dyer S, Racowsky C, de Mouzon J, Sokol $R$, et al. The international glossary on infertility and fertility care, 2017. Fertil Steril. 2017;108(3):393-406.

2. Thoma ME, Mclain AC, Louis JF, King RB, Trumble AC, Sundaram R, et al. Prevalence of infertility in the United States as estimated by the current duration approach and a traditional constructed approach. Fertil Steril. 2013; 99(5):1324-31.

3. Slama R, Hansen OK, Ducot B, Bohet A, Sorensen D, Giorgis Allemand L, et al. Estimation of the frequency of involuntary infertility on a nation-wide basis. Hum Reprod. 2012;27(5):1489-98.

4. Zhou Z, Zheng D, Wu H, Li R, Xu S, Kang Y, et al. Epidemiology of infertility in China: a population-based study. BJOG. 2018;125(4):432-41.

5. Lakatos E, Szigeti JF, Ujma PP, Sexty R, Balog P. Anxiety and depression among infertile women: a cross-sectional survey from Hungary. BMC Womens Health. 2017;17(1):48.

6. Jansen NA, Saint Onge JM. An internet forum analysis of stigma power perceptions among women seeking fertility treatment in the United States. Soc Sci Med. 2015;147:184-9.

7. Namavar Jahromi B, Mansouri M, Forouhari S, Poordast T, Salehi A. Quality of life and its influencing factors of couples referred to an infertility center in shiraz. Iran Int J Ferti Steri. 2018;11(4):293-7.

8. WHO. The World Health Organization quality of life assessment (WHOQOL): position paper from the World Health Organization. Soc Sci Med. 1995; 41(10):1403-9.

9. Namdar A, Naghizadeh MM, Zamani M, Yaghmaei F, Sameni MH. Quality of life and general health of infertile women. Health Qual Life Outcomes. 2017; 15(1):139.

10. Aduloju OP, Olaogun OD, Aduloju T. Quality of life in women of reproductive age: a comparative study of infertile and fertile women in a Nigerian tertiary Centre. J Obstet Gynaecol. 2018;38(2):247-51.

11. Masoumi SZ, Garousian M, Khani S, Oliaei SR, Shayan A. Comparison of quality of life, sexual satisfaction and marital satisfaction between fertile and infertile couples. Int J Fertil Steril. 2016;10(3):290-6.

12. Gameiro S, Boivin J, Peronace L, Verhaak CM. Why do patients discontinue fertility treatment? A systematic review of reasons and predictors of discontinuation in fertility treatment. Hum Reprod Update. 2012;18(6): 652-69.

13. Martins MV, Peterson BD, Costa P, Costa ME, Lund R, Schmidt L. Interactive effects of social support and disclosure on fertility-related stress. J Soc Pers Relat. 2013:30(4):371-88.

14. Fu B, Qin N, Cheng L, Tang G, Cao Y, Yan C, et al. Development and validation of an infertility stigma scale for Chinese women. J Psychosom Res. 2015;79(1):69-75.

15. Ganth DB, Thiyagarajan S. Nigesh. Role of infertility, emotional intelligence and resilience on marital satisfaction among Indian couples. Int J Appl Psychol. 2013;3(3):31-7.

16. Greil AL, Slauson-Blevins K, McQuillan J. The experience of infertility: a review of recent literature. Sociol Health IIIn. 2010;32(1):140-62.

17. Cserepes RE, Kollár J, Sápy T, Wischmann T, Bugán A. Effects of gender roles, child wish motives, subjective well-being, and marital adjustment on infertility-related stress: a preliminary study with a Hungarian sample of involuntary childless men and women. Arch Gynecol Obstet. 2013; 288(4):925-32

18. Kim JH, Shin HS, Yun EK. A dyadic approach to infertility stress, marital adjustment, and depression on quality of life in infertile couples. J Holist Nurs. 2018;36(1):6-14.

19. Youssef-Morgan CM, Luthans F. Psychological capital and well-being. Stress Health. 2015;31(3):180-8.

20. Martínezmartí ML, Ruch W. Character strengths predict resilience over and above positive affect, self-efficacy, optimism, social support, self-esteem, and life satisfaction. J Posit Psychol. 2016;12(2):1-10.

21. Popa-Velea O, Diaconescu L, Jidveian PM, Truțescu C. Resilience and active coping style: effects on the self-reported quality of life in cancer patients. Int J Psychiatry Med. 2017;52(2):124-36.

22. Liu L, Xu X, Xu N, Wang L. Disease activity, resilience and health-related quality of life in Chinese patients with rheumatoid arthritis: a multi-center, cross-sectional study. Health Qual Life Outcomes. 2017;15(1):149.
23. Li MY, Yang YL, Liu L, Wang L. Effects of social support, hope and resilience on quality of life among Chinese bladder cancer patients: a cross-sectional study. Health Qual Life Outcomes. 2016;14:73.

24. Herrmann D, Scherg $H$, Verres $R$, von Hagens $C$, Strowitzki T, Wischmann T. Resilience in infertile couples acts as a protective factor against infertilityspecific distress and impaired quality of life. J Assist Reprod Genet. 2011; 28(11):1111-7.

25. Wu WW, Tsai SY, Liang SY, Liu CY, Jou ST, Berry DL. The mediating role of resilience on quality of life and cancer symptom distress in adolescent patients with cancer. J Pediatr Oncol Nurs. 2015;32(5):304-13.

26. Palm-fischbacher $\mathrm{S}$, Ehlert $U$. Dispositional resilience as a moderator of the relationship between chronic stress and irregular menstrual cycle. J Psychosom Obstet Gynaecol. 2014;35(2):42-50.

27. Rainone N, Chiodi A, Lanzillo R, Magri V, Napolitano A, Morra VB, et al. Affective disorders and health-related quality of life (HRQoL) in adolescents and young adults with multiple sclerosis (MS): the moderating role of resilience. Qual Life Res. 2017;26(3):727-36.

28. Li M, Wang L. The associations of psychological stress with depressive and anxiety symptoms among Chinese bladder and renal cancer patients: the mediating role of resilience. PLoS One. 2016;11(4):e0154729.

29. Hsu PY, Lin MW, Hwang JL, Lee MS, Wu MH. The fertility quality of life (FertiQoL) questionnaire in Taiwanese infertile couples. Taiwan J Obstet Gynecol. 2013;52(2):204-9.

30. Boivin J, Takefman J, Braverman A. The fertility quality of life (FertiQoL) tool: development and general psychometric properties. Fertil Steril. 2011;96(2): 409-15.

31. Dong Y, Zhou F. Comparison of fertility quality of life between urban and rural infertile couples. Fertil Steril. 2016;106(3):e118.

32. Newton CR, Sherrard W, Glavac I. The fertility problem inventory: measuring perceived infertility-related stress. Fertil Steril. 1999;72(1):54-62.

33. Peng T, Coates R, Merriman G, Zhao Y, Maycock B. Testing the psychometric properties of mandarin version of the fertility problem inventory (M-FPI) in an infertile Chinese sample. J Psychosom Obstet Gynaecol. 2011;32(4):173-81.

34. Connor KM, Davidson JR. Development of a new resilience scale: the ConnorDavidson resilience scale (CD-RISC). Depress Anxiety. 2003;18(2):76-82.

35. Yu X, Zhang J. Factor analysis and psychometric evaluation of the ConnorDavidson resilience scale (CD-RISC) with Chinese people. Soc Behav Pers. 2007;35(1):19-30.

36. Wu L, Tan Y, Liu Y. Factor structure and psychometric evaluation of the Connor-Davidson resilience scale in a new employee population of China. BMC Psychiatry. 2017;17(1):49.

37. Karabulut A, Özkan S, Oğuz N. Predictors of fertility quality of life (FertiQoL) in infertile women: analysis of confounding factors. Eur J Obstet Gynecol Reprod Biol. 2013;170(1):193-7.

38. Aarts JW, Huppelschoten AG, van Empel IW, Boivin J, Verhaak CM, Kremer JA, et al. How patient-centred care relates to patients' quality of life and distress: a study in 427 women experiencing infertility. Hum Reprod. 2012; 27(2):488-95.

39. Santoro N, Eisenberg E, Trussell JC, Craig LB, Gracia C, Huang H, et al. Fertility-related quality of life from two RCT cohorts with infertility: unexplained infertility and polycystic ovary syndrome. Hum Reprod. 2016:31(10):2268-79.

40. Neumann K, Kayser J, Depenbusch M, Schultze-Mosgau A, Griesinger G. Can a quality-of-life assessment assist in identifying women at risk of prematurely discontinuing IVF treatment? A prospective cohort study utilizing the FertiQoL questionnaire. Arch Gynecol Obstet. 2018;298(1):223-9.

41. Casu G, Ulivi G, Zaia V, Fernandes Martins MDC, Parente Barbosa C, Gremigni P. Spirituality, infertility-related stress, and quality of life in Brazilian infertile couples: analysis using the actor-partner interdependence mediation model. Res Nurs Health. 2018:41(2):156-65.

42. Hasanpour S, Bani S, Mirghafourvand M, Yahyavi KF. Mental health and its personal and social predictors in infertile women. J Caring Sci. 2014;3(1):37-45.

43. Cheng CY, Stevenson EL, Yang CT, Liou SR. Stress and quality of life for Taiwanese women who underwent infertility treatment. J Obstet Gynecol Neonatal Nurs. 2018;47(4):498-508.

44. Lau J, Wang Q, Cheng Y, Kim JH, Yang X, Tsui HY. Infertility-related perceptions and responses and their associations with quality of life among rural Chinese infertile couples. J Sex Marital Ther. 2008;34(3):248-67.

45. Sameroff AJ, Mackenzie MJ. Research strategies for capturing transactional models of development: the limits of the possible. Dev Psychopathol. 2003; 15(3):613-40. 
46. Ju HK, Shin HS. Validation of a Korean version of fertility problem inventory. Asian Nurs Res. 2014;8(3):207-12.

47. Masoumi SZ, Khani S, Kazemi F, Kalhori F, Ebrahimi R, Roshanaei G. Effect of marital relationship enrichment program on marital satisfaction, marital intimacy, and sexual satisfaction of infertile couples. Int J Fertil Steril. 2017; 11(3):197-204

48. Pedro J, Sobral MP, Mesquita-Guimarães J, Leal C, Costa ME, Martins MV. Couples' discontinuation of fertility treatments: a longitudinal study on demographic, biomedical, and psychosocial risk factors. J Assist Reprod Genet. 2017;34(2):217-24.

49. Moura-Ramos M, Gameiro S, Canavarro MC, Soares I. Assessing infertility stress: re-examining the factor structure of the fertility problem inventory. Hum Reprod. 2012;27(2):496-505.

50. Jisha PR, Thomas I. Quality of life and infertility: influence of gender, years of marital life, resilience, and anxiety. Psychol Stud. 2016;61(3):159-69.

51. Martin PR. Stress and primary headache: review of the research and clinical management. Curr Pain Headache Rep. 2016;20(7):45.

52. Peterson B, Boivin J, Norré J, Smith C, Thorn P, Wischmann T. An introduction to infertility counseling: a guide for mental health and medical professionals. J Assist Reprod Genet. 2012;29(3):243-8.

53. Johnston MC, Porteous T, Crilly MA, Burton CD, Elliott A, Iversen L, et al. Physical disease and resilient outcomes: a systematic review of resilience definitions and study methods. Psychosomatics. 2015;56(2):168-80.

54. Stonnington CM, Darby B, Santucci A, et al. A resilience intervention involving mindfulness training for transplant patients and their caregivers. Clin Transpl. 2016;30(11):1466-72.

55. Galante J, Dufour G, Vainre M, et al. A mindfulness-based intervention to increase resilience to stress in university students (the mindful student study): a pragmatic randomised controlled trial. Lancet Public Health. 2018; 3(2):e72.

56. Zalta AK, Tirone V, Siedjak J, et al. A pilot study of tailored cognitive-behavioral resilience training for trauma survivors with subthreshold distress. J Trauma Stress. 2016;29(3):268-72.

57. Schmidt M, Haglund K. Debrief in emergency departments to improve compassion fatigue and promote resiliency. J Trauma Nurs. 2017;24(5): $317-22$.

Ready to submit your research? Choose BMC and benefit from:

- fast, convenient online submission

- thorough peer review by experienced researchers in your field

- rapid publication on acceptance

- support for research data, including large and complex data types

- gold Open Access which fosters wider collaboration and increased citations

- maximum visibility for your research: over $100 \mathrm{M}$ website views per year

At $\mathrm{BMC}$, research is always in progress.

Learn more biomedcentral.com/submissions 